\title{
Hydrometrocolpos, postaxial polydactyly, and hypothalamic hamartoma in a patient with confirmed Pallister-Hall syndrome: a clinical overlap with McKusick-Kaufman syndrome
}

\author{
Sebastian Kos $\cdot$ Katharina Roth $\cdot$ Dirk Korinth $\cdot$ \\ Georg Zeilinger • Georg Eich
}

Received: 12 February 2008 /Revised: 25 March 2008 / Accepted: 1 April 2008 / Published online: 14 May 2008

(C) Springer-Verlag 2008

\begin{abstract}
We present a preterm-born girl with polydactyly of both hands and massive hydrometrocolpos, the latter due to vaginal atresia. This association led initially to the diagnosis of McKusick-Kaufman syndrome (MKKS). However, additional features, including characteristic radiographic findings of the hands and a large hypothalamic tumour, presumably a hamartoma, favoured the diagnosis of Pallister-Hall syndrome (PHS), which was then genetically confirmed by detection of a GLI3 mutation (Q717X). This is the second genetically confirmed case revealing the previously described association of PHS with hydrometrocolpos due to vaginal atresia as a clinical overlap with MKKS.
\end{abstract}

Keywords Pallister-Hall · McKusick-Kaufman . Hydrometrocolpos $\cdot$ Child

\author{
S. $\operatorname{Kos}(\bowtie)$ \\ Institute of Radiology, University Hospital Basel, \\ Petersgraben 4, \\ 4031 Basel, Switzerland \\ e-mail: skos@gmx.de \\ K. Roth \\ Division of Pediatric Surgery, Kantonsspital Aarau, \\ Aarau, Switzerland \\ D. Korinth \\ Institute of Medical Genetics, University Zürich, \\ Zürich, Switzerland \\ G. Zeilinger \\ Department of Pediatrics, Kantonsspital Aarau, \\ Aarau, Switzerland \\ G. Eich \\ Department of Radiology, Kantonsspital Aarau, \\ Aarau, Switzerland
}

\section{Introduction}

In 1980, Hall et al. [1] described a partly lethal syndrome (Pallister-Hall syndrome, PHS; MIM 146510) in six infants and neonates. The malformations included postaxial polydactyly, hypothalamic hamartoma, imperforate anus, intrauterine growth retardation and several visceral anomalies, including renal agenesis or dysplasia, renal ectopia, congenital heart defects (persistent ductus arteriosus, ventricular septal defect, endocardial cushion defect, mitral and aortic valve defects and aortic coarctation), microphallus, cryptorchidism, and abnormal lung lobation. PHS is inherited with an autosomal-dominant trait with variable expressivity [2]. Mutations in the zinc-finger transcription factor gene (GLI3) on the chromosomal locus $7 \mathrm{p} 13$ were found to cause PHS in 2005 [3].

McKusick-Kaufman syndrome (MKKS), characterized by hydrometrocolpos due to vaginal atresia, imperforate anus, postaxial polydactyly and congenital heart defects, may present with clinical features similar to those of PHS. So far, only one case of PHS with hydrometrocolpos has been genetically proven; another one was suspected to represent PHS $[4,5]$. The patient with genetically confirmed PHS had a c.2567 C>A mutation. However, no hydronephrosis or congenital heart defect, as in our patient, was present (Table 1). Therefore, our patient further enlarges the spectrum of associated malformations in PHS.

\section{Case report}

This infant was born to nonconsanguineous healthy parents. The mother $(\mathrm{G} 3, \mathrm{P} 3)$ was 40 years of age and the father was 36 years of age. The pregnancy was uneventful without 
Table 1 Summary of the features of PHS and MKKS compared with those of the patients reported by Unsinn et al. [4] and McCann et al. [5] and our patient

\begin{tabular}{lccccc}
\hline Features & PHS & MKKS & Reference 4 & Reference 5 & Our patient \\
\hline Hydrometrocolpos & - & + & + & + & + \\
Postaxial polydactyly & + & + & + & + & + \\
Hamartoma & + & - & + & + & + \\
Nail dysplasia & + & + & + & + & + \\
Congenital heart defects & + & + & Not investigated & + & + \\
Hydronephrosis & + & - & + & + \\
Facial dysmorphism & + & - & + & + \\
GLI 3 mutation & & & & + & + \\
\hline
\end{tabular}

recorded drug exposure, infection, or unusual environmental factors. On routine US at 34 weeks a large cystic mass was found in the fetal abdomen. Amniocentesis revealed a normal female karyotype 46,XX. The increasing size of the cyst on repeat examination led to early delivery by caesarean section at $36^{+0}$ weeks. Birth weight was $2,700 \mathrm{~g}$ (25th to 50th centile) with a length of $44.5 \mathrm{~cm}$ (3rd to 10th centile) and a head circumference of $31 \mathrm{~cm}$ (3rd to 10th centile). The APGAR score was 3/6/7. The newborn was intubated due to respiratory insufficiency.

The initial babygram showed a large abdominal mass displacing the intestine and elevating the diaphragms (Fig. 1). Abdominal US revealed a fluid-filled and massively dilated vagina and uterus (hydrometrocolpos) that compressed and displaced the urinary bladder anteriorly (Fig. 2). The kidneys were small, the long axis being $3.0 \mathrm{~cm}$ on the left and $2.4 \mathrm{~cm}$ on the right. Dilatation of the ureters and collecting systems was present. Serum creatinine $(184 \mu \mathrm{mol} / \mathrm{l})$ and urea $(9.7 \mathrm{mmol} / \mathrm{l})$ were elevated. A bladder catheter was inserted through the single introital orifice. Percutaneous insertion of another catheter led to drainage of urine from the vagina. Contrast studies of the urinary bladder and vagina (Fig. 2) and vesicoscopy confirmed a fistula between the bladder neck and vagina.

Dysmorphic features at the age of 3 months included microcephaly, small nose (Fig. 3), high-arched palate, brachydactyly of the hands and feet, and a sacral dimple. Postaxial heptadactyly and brachydactyly of both hands were present. In addition, hypoplasia of the finger nails and single palmar creases were noted (Fig. 3).

Cranial US, undertaken because of the suspicion of PHS, revealed a large hypothalamic mass (Fig. 4). MRI (Fig. 4) confirmed the presence and location of the mass. It was solitary, nonenhancing, and isointense to grey and white matter of the brain, consistent with a hamartoma.

Neurological, ophthalmological and otological examinations were unremarkable. Apart from isolated deficiency of growth hormone $(\mathrm{GH})$, no hypopituitarism or adrenal insufficiency was found. Echocardiography showed mild tricuspid regurgitation, a small patent foramen ovale, and a persistent ductus arteriosus with resulting left-to-right shunt.

\section{Genetic analysis}

Consecutive gene analysis was performed by the Institute for Molecular and Human Genetics of the Georgetown University Medical Center in cooperation with the team of L. Biesecker at the National Human Genome Research

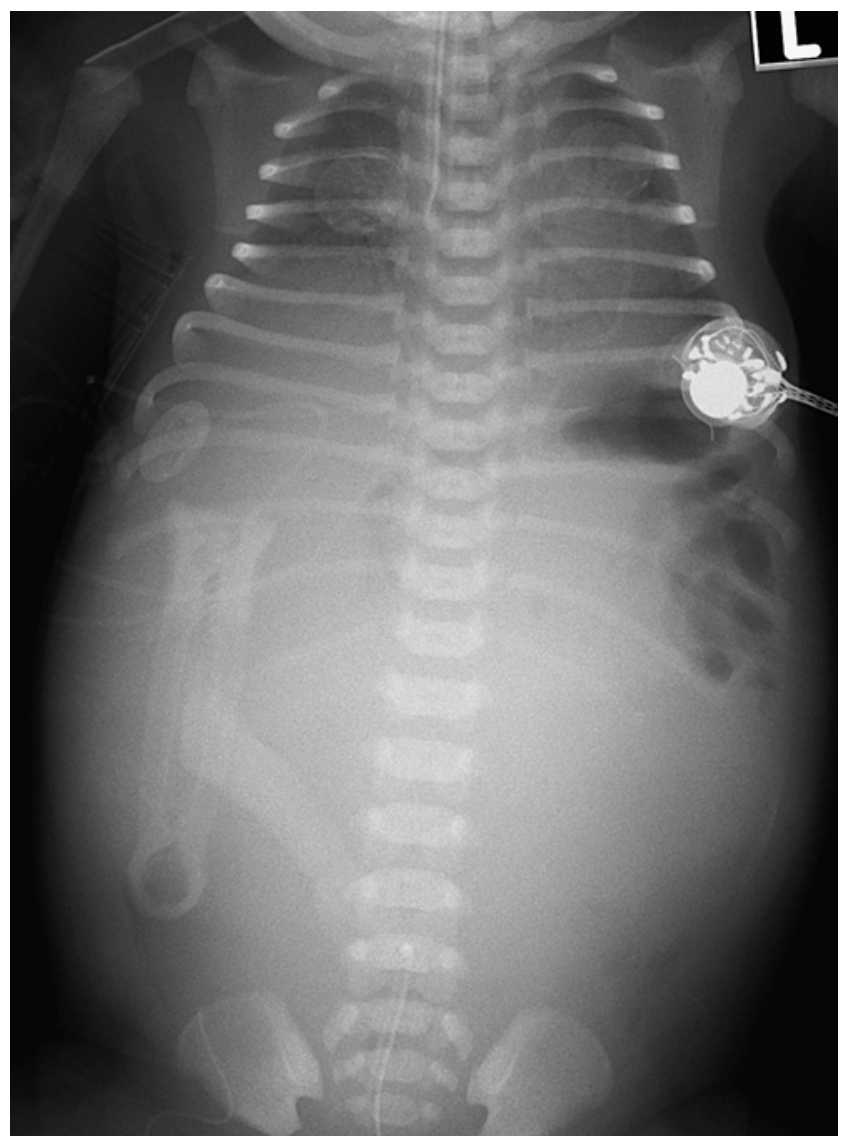

Fig. 1 Initial babygram at birth following tracheal intubation shows a large lower abdominal mass that displaces the intestine superiorly and laterally and elevates the diaphragms. No calcifications are present 


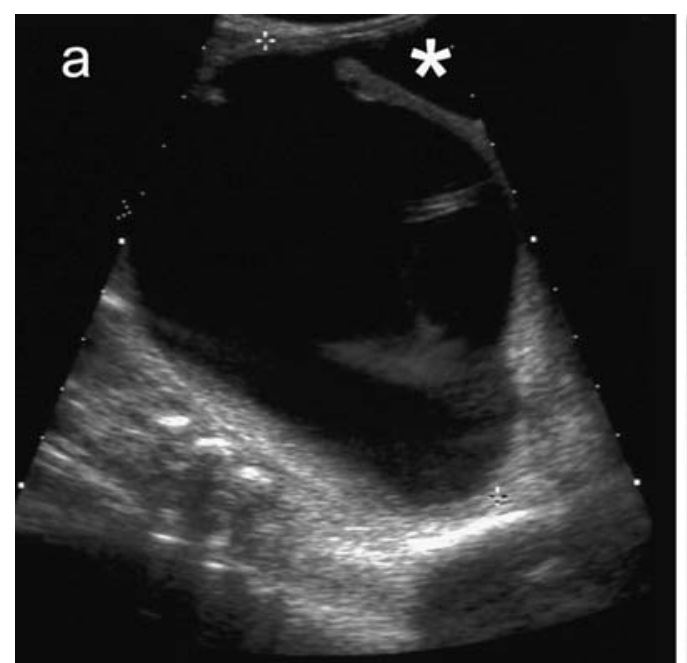

Fig. 2 Imaging of the urogenital tract. a Sagittal US image of the lower abdomen shows the fluid-filled vagina and uterine cavity (asterisk). b Contrast medium injected into the bladder through the indwelling catheter demonstrates an anteriorly displaced and compressed urinary bladder and faint opacification of the dilated vagina

Institute in Bethesda, MD, USA. PCR amplification of exon 14 of GLI3 gene and its flanking intronic region (forward np 11,602-11,622, and reverse np 12,065-12,084; forward np 11,611-11,630, and reverse np 12,019-12,041
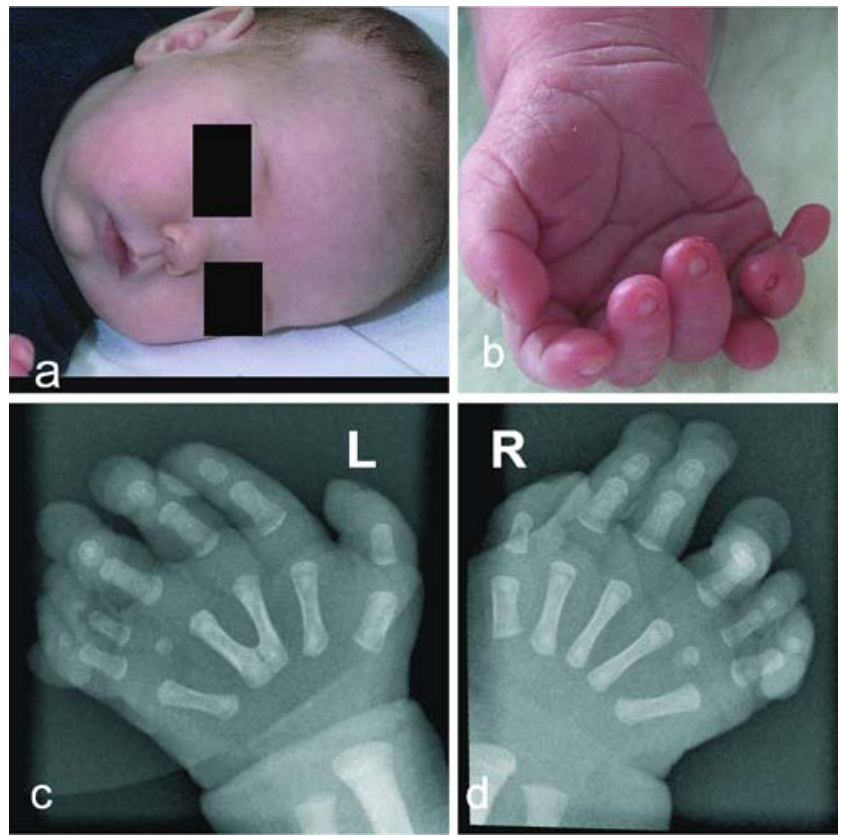

Fig. 3 Facial and hand appearances. a Facial photograph. Note small nose, depressed nasal bridge and long philtrum. b Photograph of the right hand shows postaxial polydactyly with seven digits. Note hypoplastic finger nails and a single palmar crease. Radiographs of the left hand (c) and right hand (d) show seven digits and six metacarpals. The first and particularly the distally displaced fifth metacarpals are short with a proximal defect of the fifth. There is partial fusion of the left third and fourth metacarpals that appear Vshaped. The middle phalanges are short and oval shaped. Absent ossification of the distal phalanges is noted
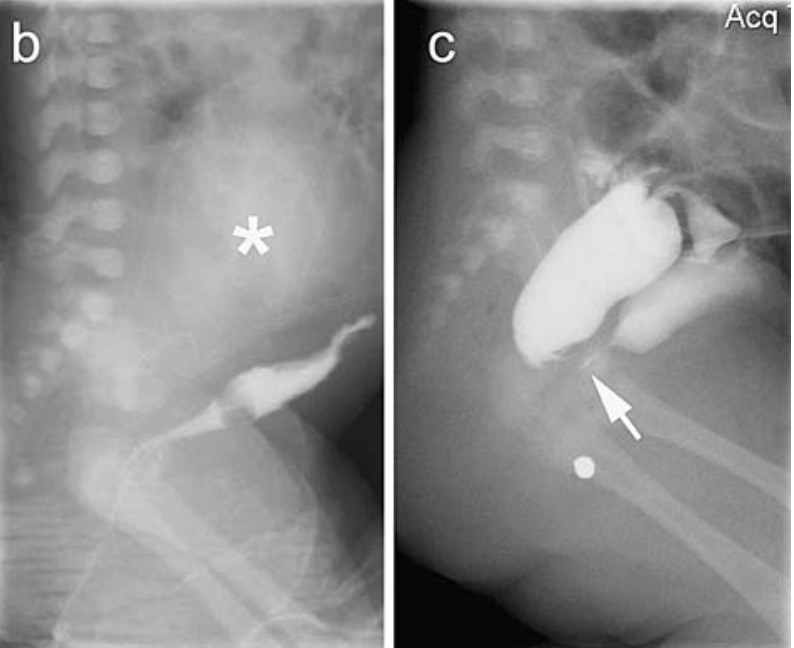

posteriorly (asterisk). c Contrast medium injected into the vagina through the vaginostomy catheter shows opacification of the urinary bladder via a small fistula (arrow) between the vagina and urethra. Note the lead marker at the urethral opening

primers) followed by direct DNA sequencing with forward primers (np 11,602-11,622 and np 11,611-11,630) confirmed a mutation at c.2149C $>$ T (Q717X). This mutation was detected only in the index patient and not in her parents, and most likely represented a de novo mutation. A germ-cell mutation mosaic cannot be ruled out, hitherto described in only one case [6].

\section{Discussion}

The association of hydrometrocolpos and postaxial polydactyly led to the initial differential diagnosis of BardetBiedel syndrome (BBS), MKKS and PHS (Table 2) [7, 8]. However, the peculiar bone morphology of the hands matched the original description of PHS and was less compatible with either MKKS or BBS.

The subsequent search for the hypothalamic hamartoma confirmed a hypothalamic and suprasellar lesion that was isoechoic and isointense to normal brain tissue, was nonenhancing and, therefore, compatible with a hamartoma. Hypothalamic hamartoma is a rare usually isolated lesion, but a major feature of PHS. An association with BBS is very rare and not known with MKKS. Other features of our patient are consistent with PHS and inconsistent with either MKKS or BBS (Table 2).

Familial PHS is known to be inherited as an autosomaldominant trait with variable expressivity. Linkage with mutations of the zinc-finger transcription factor (GLI3) on 7 p13 has been demonstrated in some of these patients. GLI3 mutations were present in $95 \%$ of all patients with clinically diagnosed PHS [3]. Interestingly, mutations in the 

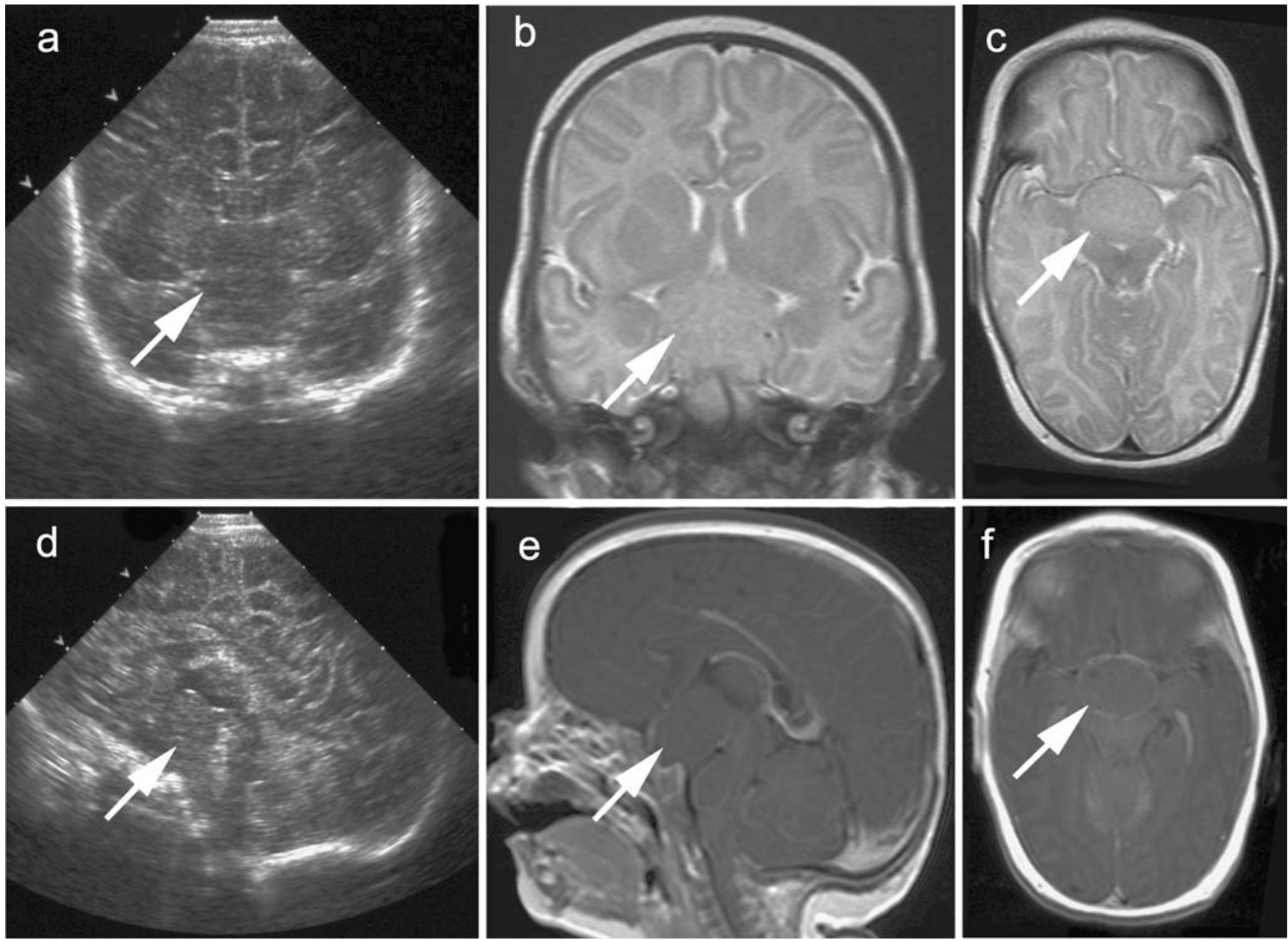

Fig. 4 Hypothalamic hamartoma. a, d Cranial US images (a coronal, d sagittal) show a sharply marginated hypothalamic and suprasellar mass lesion (arrow) with a diameter of approximately $2.5 \mathrm{~cm}$. The mass is homogeneous and isoechoic with the brain. Hydrocephalus is not present. b, c, e, f Cranial MR images (b coronal T2-W, c axial T2-

W, e sagittal contrast-enhanced T1-W, f axial contrast-enhanced T1W) show the hypothalamic mass (arrow) to be nonenhancing and isointense to grey and white matter in all sequences, consistent with a hamartoma

GLI3 gene are also responsible for the Greig cephalopolysyndactyly syndrome (GCPS) with two clear phenotypephenotype correlations [3]. PHS is caused by frameshift/ nonsense and splicing mutations, but many types of alterations in the GLI3 gene cause GCPS, i.e. trans-

locations, large deletions, exonic deletions and duplications and small in-frame deletions, and missense, frameshift/ nonsense, and splicing mutations. Mutations in the first third of the GLI3 gene cause PHS, whereas mutations in the second and third thirds are responsible for GCPS.

Table 2 Key features of MKKS, BBS and PHS

\section{Syndrome Features}

MKKS An autosomal recessive disorder that typically presents with hydrometrocolpos in infant girls and postaxial polydactyly. MKKS may be associated with hydronephrosis, Hirschsprung disease, and imperforate anus. It is caused by a mutation in the MKKS gene (OMIM, GeneTests).

BBS An autosomal recessive disorder with features overlapping with those of MKKS including polydactyly and urogenital anomalies. Other major features include: rod-cone dystrophy, hypogonadism, obesity, and learning disabilities. Mutations of the BBS1BBS10 genes and the MKKS gene are known to cause BBS (OMIM, GeneTests).

PHS An autosomal dominant disorder characterized by hypothalamic hamartoma, central and postaxial polydactyly, congenital heart defects, nail hypoplasia, facial dysmorphism, and imperforate anus. Mutations of the GLI3 gene cause PHS (OMIM, GeneTests). 
PHS caused by the GLI3 mutation (Q717X) identified in our patient has been described in only one previous patient [5]. Corresponding clinical manifestations in our patient were hypothalamic hamartoma, insertional and postaxial polydactyly, and nail hypoplasia. However, our patient did not show a bifid epiglottis, dental hypoplasia, imperforate anus and (at the time of this report) no seizures, whereas the genitourinary malformations were not seen in the previously described patient, suggesting that there is no genotypephenotype correlation in PHS.

As the GLI3 mutation (Q717X) was detected in our patient, but not in her parents, it is most likely to have been a de novo mutation. As there has been only one previously described patient with germ-cell mosaic [6], its presence in the parents and the likelihood of recurrence in prospective offspring is very low $(<1 \%)$.

In conclusion, we present the second genetically confirmed association of PHS with hydrometrocolpos due to vaginal atresia. In addition we demonstrate that this association may also include congenital heart defects and hydronephrosis as described above, which were not detected in the patient described by McCann et al. [5]. Therefore, hydrometrocolpos due to vaginal atresia is an overlapping feature of PHS and MKKS.

\section{References}

1. Hall JG, Pallister PD, Clarren SK et al (1980) Congenital hypothalamic hamartoblastoma, hypopituitarism, imperforate anus and postaxial polydactyly - a new syndrome? Part I: clinical, causal, and pathogenetic considerations. Am J Med Genet 7:47-74

2. Topf KF, Kletter GB, Kelch RP et al (1993) Autosomal dominant transmission of the Pallister-Hall syndrome. J Pediatr 123:943-946

3. Johnston JJ, Olivos-Glander I, Killoran C et al (2005) Molecular and clinical analyses of Greig cephalopolysyndactyly and PallisterHall syndromes: robust phenotype prediction from the type and position of GLI3 mutations. Am J Hum Genet 76:609-622

4. Unsinn KM, Neu N, Krejci A et al (1995) Pallister-Hall syndrome and McKusick-Kaufmann syndrome: one entity? J Med Genet 32:125-128

5. McCann E, Fryer AE, Craigie R et al (2006) Genitourinary malformations as a feature of the Pallister-Hall syndrome. Clin Dysmorphol 15:75-79

6. Ng D, Johnston JJ, Turner JT et al (2004) Gonadal mosaicism in severe Pallister-Hall syndrome. Am J Med Genet A 124:296-302

7. GeneTests (2008) Medical genetics information resource (online database). Copyright University of Washington, Seattle. Updated weekly. http://www.genetests.org. Accessed 14 April 2008

8. Online Mendelian Inheritance in Man, OMIM (2008) McKusickNathans Institute of Genetic Medicine, Johns Hopkins University (Baltimore, MD) and National Center for Biotechnology Information, National Library of Medicine (Bethesda, MD). http://www. ncbi.nlm.nih.gov/omim/. Accessed 17 Apr 2008 